\title{
INFLUENCIA DE LOS PROCESOS DE PIPING EN LA EVOLUCIÓN DEL MODELADO. BARDENAS REALES (NAVARRA, ESPAÑA)
}

\author{
G. DESIR* y C. MARÍN \\ Departamento de Ciencias de la Tierra. \\ Facultad de Ciencias. Universidad de Zaragoza. \\ Pedro Cerbuna, 12, 50.009 Zaragoza.
}

RESUMEN. Los procesos de piping están controlados por un gran número de factores que interactúan entre sí, como son el índice de dispersión y el contenido en sodio, SAR y ESP. A ello hay que unir la densidad de agrietamiento y la pendiente de las laderas sobre las que se desarrolla. En el área de estudio las características climáticas junto con la estructura del suelo y la composición química y mineralógica del sustrato tienen un papel esencial en la formación de piping. El agrietamiento va ligado a la presencia de arcillas dispersivas con elevados contenidos en SAR y ESP, que confieren al sustrato unas tasas de hinchamiento de hasta el $12 \%$.

La presencia de procesos de piping está ligada a dos situaciones concretas: los niveles masivos intermedios del relleno holoceno de la cuenca y los sedimentos depositados por los gullies; mientras que en los materiales terciarios no se llega a desarrollar. La dimensión y magnitud de los pipes analizados, muestran una clara relación entre la potencia del nivel, la pendiente y el gradiente hidráulico. En los rellenos el principal factor limitante es la potencia pues los niveles laminares inferiores no facilitan la continuidad en profundidad del proceso debido al elevado número de discontinuidades generando conductos de escala centimétrica que siempre están sobre impuestos a la red de drenaje. En los sedimentos de los gullies el tamaño y diámetro de los pipes es función directa de las dimensiones del gully e inversamente proporcional a la proximidad al borde.

Influence of piping processes on the relief evolution. Bardenas Reales (Navarra, Spain)

ABSTRACT. Piping processes are controlled by many interacting factors, such as dispersion index and sodium content, SAR and ESP, together with other important factors as cracking density, slope degree and climate. In the study area 
piping is linked to poor developed soils with high sodium contents which give raise to an intense regolith cracking that favours infiltration and finally piping. As the results show piping in this area can be separated in two different types depending on its genesis, one based on seepage processes, considered as a true type, and another due to tunnelling processes. From a distributional point of view piping is linked to two concrete situations: the massive intermediate levels of the intermediate Holocene filling sediments and the sediments deposited by gullies. In the tertiary materials, in general, it does not manage to develop. On Holocene materials pipes size and scale show a clear relationship among the level thickness, slope and hydraulic gradient, being the first one the principal bounding factor. Pipes developed on the lower laminated levels do not reach important depth due to the high number of discontinuities, generating centimetre-scale conduits that are always on duty to the drainage network. On gullies sediments piping are related to gullies margin evolution and headcut retreat. Pipes size and diameter are a direct function of gully dimensions and are inversely proportional to the proximity to gully scarp. Pipes are on a metric scale with a vertical pattern which development in depth is controlled by upper level thickness, since they are circumscribe to him.

Palabras clave: piping, gully erosion, arcillas, propiedades físico-químicas, suelos dispersivos.

Key words: piping, gullies, dispersive clays, physic-chemical properties.

Enviado el 14 de octubre de 2010

Aceptado el 7 de diciembre de 2010

*Correspondencia: Departamento de Ciencias de la Tierra. Facultad de Ciencias. Universidad de Zaragoza. Pedro Cerbuna, 12, 50009 Zaragoza. E. mail: gdesir@unizar.es

\section{Introducción}

La intensidad y el grado de desarrollo del piping en un área son reflejo de la interacción entre las variables climáticas, las propiedades del regolito y el gradiente hidráulico local (Bryan y Jones, 1997). Cuando nos encontramos en zonas donde el gradiente es bajo y la tasa de infiltración pequeña es difícil que se desarrollen pipes debido principalmente a la ausencia de dicho gradiente y de un mecanismo que concentre el agua infiltrada (Faulkner et al., 2000). En áreas constituidas básicamente por materiales arcillosos de bajo o nula permeabilidad, como es el área de este estudio, el piping solo se desarrolla ligado a regolitos con elevados contenidos en arcillas sódicas y en zonas próximas a los márgenes de los barrancos o gullies (Desir et al., 2005; Desir y Marín, 2006). En ambas situaciones la infiltración de la escorrentía tiene lugar a través de las grietas (mudcracks), cuyo desarrollo está controlado por las propiedades físico-químicas del material y las características climáticas de la zona. La presencia de una precipitación 
de tipo estacional, muy variable, hace que se produzca el agrietamiento del regolito durante los periodos secos constituyendo la principal vía de penetración del agua de escorrentía en el regolito al tratarse de formaciones arcillosas altamente impermeables. Y está ligado a la presencia de arcillas hinchables del tipo montmorillonita o arcillas con elevados contenidos en sodio intercambiable. En el área de estudio el agrietamiento está condicionado por la presencia de arcillas con elevados contenidos en sodio intercambiable, SAR y ESP, que confieren al sustrato unas tasas de hinchamiento de hasta el $12 \%$ y elevados coeficientes de dispersión (Gutiérrez et al., 1995). Diversos autores (Jones, 1981; Martínez-Casanovas et al., 2004) señalan la dispersión de las arcillas como una de los mecanismos más importantes en el proceso de piping.

El objetivo de este trabajo es conocer cuáles son los factores principales que controlan la aparición y el desarrollo de los procesos de piping en esta área. Para ello se analizaran las diferentes situaciones y factores que controlan su aparición y las características de los pipes y de de las formaciones sobre las que aparecen.

\section{2. Área de estudio}

Las Bardenas Reales, sector centro-occidental de la Depresión del Ebro, constituyen una depresión erosiva de $415 \mathrm{~km}^{2}$ de extensión, formada por sedimentos de edad Miocena y Cuaternaria (Fig.1). Los sedimentos terciarios, arcillas miocenas de la Formación Tudela, aparecen en los márgenes de la cuenca dando lugar a pendientes escarpadas con una alta densidad de reguerización. Los depósitos cuaternarios, arcillas y limos holocenos procedentes del lavado de las arcillas circundantes, han sufrido una intensa erosión y el desarrollo de profundos gullies sobre ellos. El clima es semiárido de carácter mediterráneo, con una temperatura media anual de $13^{\circ} \mathrm{C}$ y una precipitación media anual de $350 \mathrm{~mm}$. Las lluvias son de carácter tormentoso con una gran variabilidad estacional e intensidad

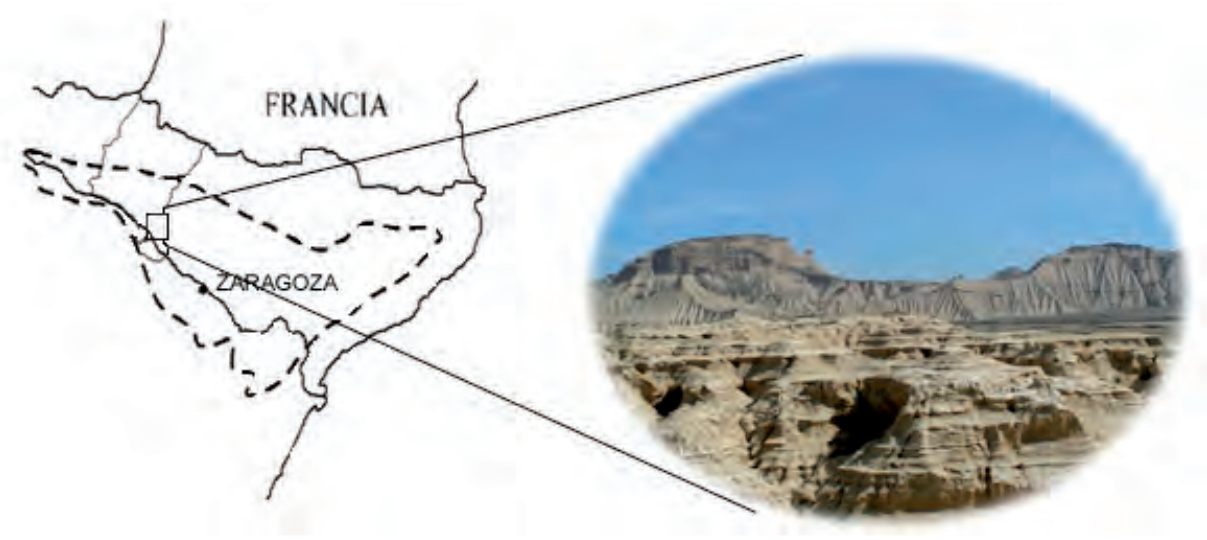

Figura 1. Localización del área de estudio. 
Los materiales terciarios de la Fm. Tudela son arcillas con altos contenidos en sodio, SAR y ESP, por encima de 25 y 30 respectivamente, $\mathrm{pH}$ alcalinos y elevadas conductividades eléctricas. Mineralógicamente, está compuesta por minerales de la arcilla (illita, 82-86\%, clorita, 9-16\%, e indicios de caolinita), cuarzo y calcita. Todas estas propiedades les confieren una capacidad de hinchamiento que puede llegar a superar el $12 \%$ e índices de dispersión en torno a 0.5 a 0.8 (Gutiérrez et al., 1995, 1997). El índice de plasticidad se encuentra entre 5,0 y 18,7 lo que indica que el tránsito entre el comportamiento plástico y el líquido es muy rápido (Gutiérrez et al., 1995).

Los materiales holocenos son arcillas y limos provenientes del lavado de las arcillas terciarias circundantes de la Fm. Tudela (Gutiérrez et al., 1995). Se trata de un material poco consolidado que se encuentra fuertemente incidido (Desir et al., 2005) y que está compuesto por tres niveles principales, dos niveles laminares separados por otro masivo más arcilloso. La potencia del regolito en los tres niveles es inferior a 3-4 cm. Son arcillas con elevados índices de dispersión ( 0.6 a 0.7 niveles superiores y superior a 0.9 el nivel basal), y porcentajes de hinchamiento que oscilan entre 4,3 y 4,5\% (Gutiérrez et al., 1995). Desde un punto de vista químico, se trata de unas arcillas con $\mathrm{pH}$ débilmente alcalino, conductividad eléctrica elevada y altos valores de SAR y ESP, por encima de 30 en ambos casos. Mineralógicamente, están constituidos básicamente por minerales de la arcilla (illita, $81-85 \%$, clorita, 11-17\%, y trazas de caolinita).

\section{Metodología}

Para analizar el proceso de piping en la zona de estudio y cuáles son los factores que lo controlan se seleccionaron tres zonas diferentes en las que se presenta el piping. La primera zona corresponde a los depósitos del rellenos de la cuenca en los márgenes de un gully que ha evolucionado a partir de una zanja de desagüe de una de las parcelas experimentales para el estudio de la erosión (Desir y Marín, 2006). En esta zona se han medido y analizado un total de 22 pipes. La segunda zona se sitúa próxima a la anterior pero se asienta sobre materiales holocenos laminados de depósito de gullies próxima al margen de un gully de grandes proporciones, $25 \mathrm{~m}$ de ancho por 8-10 $\mathrm{m}$ de profundidad. Finalmente, la tercera zona se encuentra en los márgenes de un pequeño relieve tabular, Cabecico Losado, desarrollado sobre los materiales holocenos del relleno general de la cuenca. En esta zona se han analizado un total de 25 pipes.

En cada una de estas zonas se midieron la anchura, longitud, profundidad de cada uno de los pipes, así como la distancia al vecino más próximo y la distancia al gully. También se midió la altura relativa del outlet sobre el fondo del gully, la potencia del nivel sobre el que se desarrollan y el tipo de grietas precursoras. Finalmente, y para conocer cuáles son los factores que controlan este proceso se han analizado las propiedades físico-químicas de las diferentes formaciones sobre las que se desarrollan los procesos de piping. 


\section{Resultados y discusión}

En nuestra área de estudio el piping aparece representado como uno de los principales procesos de erosión, ligado a procesos de reguerización y abarrancamiento de los materiales terciarios y cuaternarios que rellenan la Depresión erosiva de las Bardenas Reales (Desir et al., 2005; Desir y Marín, 2006). Ello se debe básicamente a la combinación de varios factores como son la presencia de lluvias con una gran variabilidad estacional, materiales con elevadas contenidos en SAR, elevado $\mathrm{pH}$ y especialmente a la presencia de materiales con capacidad de hinchamiento (Gutiérrez et al., 1995).

La presencia de procesos de piping en el área de estudio está ligada a dos situaciones concretas: los rellenos holocenos de la cuenca, especialmente al nivel masivo intermedio y a los sedimentos depositados por los gullies. En los materiales terciarios no se llega a desarrollar piping en ninguno de los niveles reconocidos, a pesar que los resultados de la analítica realizada muestran valores de SAR y ESP que indican lo contrario (Gutiérrez et al., 1995). En este caso el piping se ve inhibido por la elevada pendiente de las laderas, por encima de $30^{\circ}$, la escasa penetración del sistema de grietas en profundidad y la reducida potencia del regolito, $2-5 \mathrm{~cm}$, que impiden que el agua de escorrentía se infiltre y profundice más allá del este nivel de alteración. Ello favorece el desarrollo en épocas frías y húmedas de movimientos de masas tipo colada de barro (mud slides) que inhiben el desarrollo del piping al remover los pipes incipientes y borrar los posibles puntos en los cuales se puede desencadenar el proceso.

\subsection{Clasificación del piping en función del mecanismo de formación}

Dos tipos de piping: uno que se ajusta al proceso de seepage; comienza en las partes bajas y se propaga hacia la parte superior mediante la ampliación de los conductos y la erosión remontante. Y otro asociado a procesos de tunneling, en el que la erosión y la formación de los conductos progresa a través de las grietas superficiales hacia el interior. En ambos casos las grietas se pueden formar por dos mecanismos diferentes, como consecuencia de los proceso de contracción y dilatación y la presencia de minerales de la arcilla hinchables o bien por esfuerzos mecánicos, donde la presencia de un gully cercano y la evolución de los márgenes genera grietas de extensión que son las precursoras de los pipes. Cada uno de estos mecanismos está directamente relacionado con el mecanismo generador de pipes, de manera que los pipes formados por seepage responden al primer tipo de agrietamiento y los formados por tunneling al segundo.

En función del tipo de agrietamiento los pipes del área de estudio se pueden clasificar en:

- Pipes asociados a grietas de desecación. Micro Pipes (seepage) (Fig.2). Más que pipes apertura de grietas que favorecen la meteorización y los movimientos de masas. 

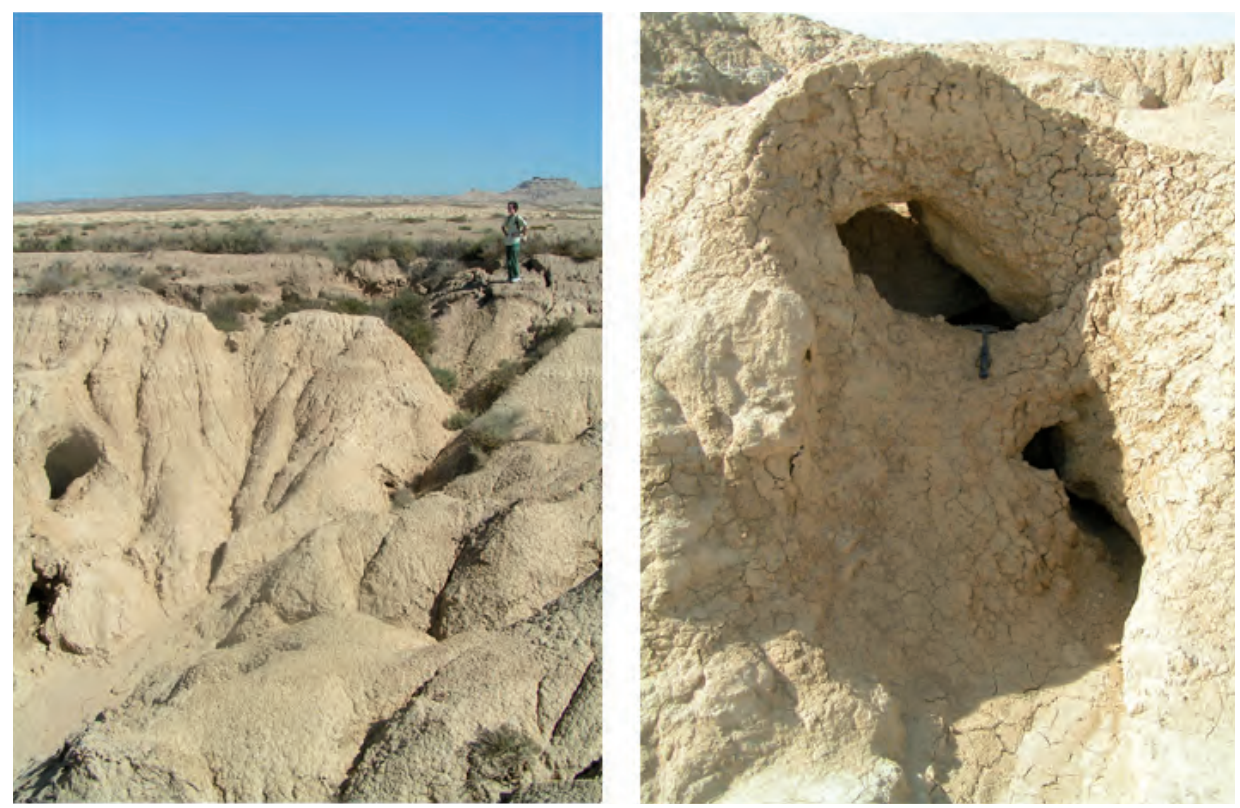

Figura 2. Dos aspectos en campo de los pipes desarrrollados por procesos de seepage en las formaciones arcillosas de los rellenos de la cuenca.
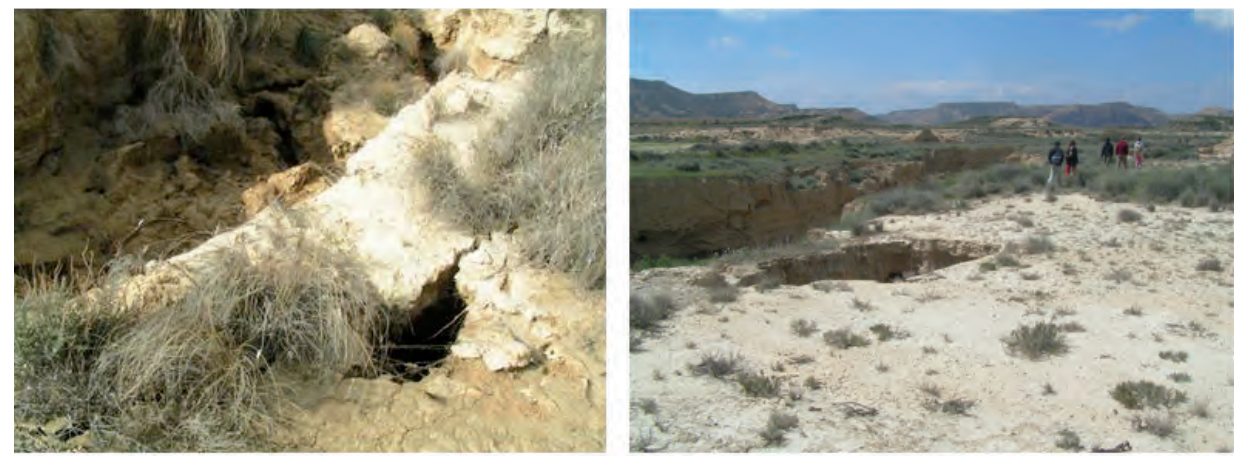

Figura 3. A) Detalle de la grieta extensional en el márgen de un gully e inicio de un pequeño pipe incipiente y $B$ ) pipe de gran tamaño formado en el margen del barranco principal.

- Asociados a grietas de extensión. Gully Pipes (tunnelling) (Fig.3).Colapsos circulares o pseudokarst se producen en la época húmeda debido al aumento del peso y a la pérdida de cohesión por el incremento de la humedad. Pipes de grandes dimensiones que se desarrollan en los márgenes de los grandes gullies o de los escarpes estructurales mayores y llegan a afectar al sustrato terciario. Son semejantes a los descritos por Jones (1981). 

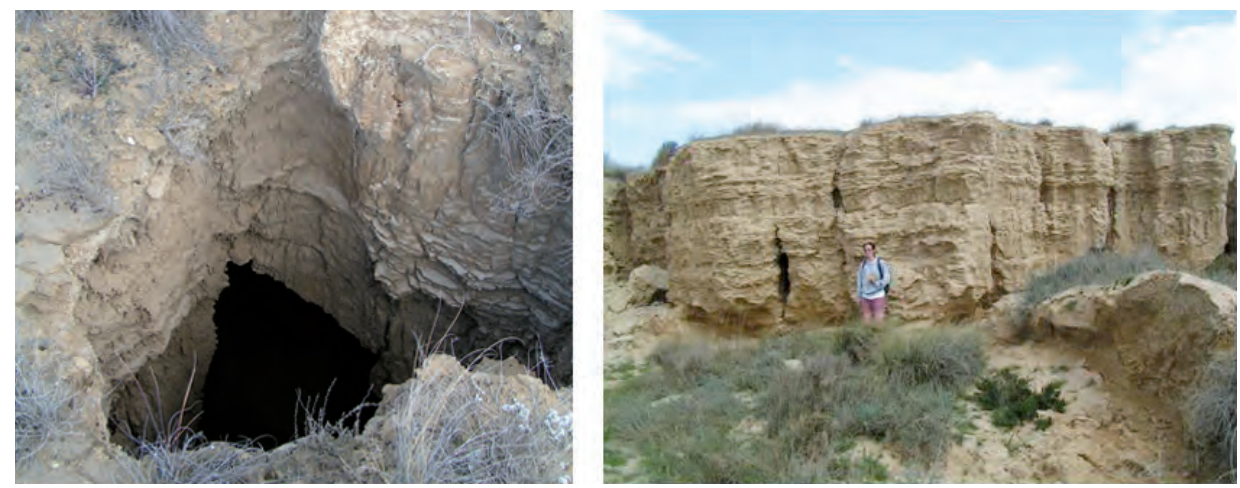

Figura 4. A) Vista somital de un pipe generado por procesos de seepage en el margen de un gully. En la imagen se puede observar como el fondo del pipe coincide con el techo del nivel infrayacente. B) Vista lateral de estos pipes con conductos verticalizados.

- Pipes asociados a contactos litológicos. Gully Pipes (seepage) (Fig. 4). Se producen en gullies nuevos con conductos verticales hasta el contacto con otra formación que actúa como nivel impermeable a partir del cual el conducto se desarrolla horizontalmente. Se pueden asociar a los pozos de Gracia (1986) y Gallart (1979), cuando el gully no profundiza hasta el nivel masivo inferior.

\subsection{Distribución espacial y tipos de piping}

Gully vs. Piping. En las Bardenas Reales predominan los pipes con conductos verticales que están asociados a las facies de los depósitos de gullies (ripples) y se restringen a los márgenes de los barrancos. Son equivalentes a los pozos de Gracia (1986). Estos pipes son función directa de las dimensiones del gully e inversamente proporcional a la proximidad al borde. Son conductos de escala métrica a decamétrica con un trazado netamente vertical cuyo desarrollo en profundidad está controlado por la potencia del nivel superior del relleno, pues se circunscriben a él. Cuando alcanzan este nivel evolucionan mediante la ampliación del conducto. El nivel inferior del relleno de los gullies actúa como nivel impermeable de modo que los pipes siguen este trazado con una trama horizontal hasta interceptar el gully (Fig.4). En los gullies mayores pueden sobrepasarse estos niveles y afectar a los sedimentos terciarios infrayacentes (Fig.5). Estudios previos realizados sobre un gully generado antrópicamente corroboran las pautas de evolución observada en estos gullies, donde el desarrollo de piping actúa como precursor facilitando en primera instancia la ampliación del canal y el retroceso de las cabeceras (Desir y Marín, 2004). Se forman por proceso de see page, es decir desde arriba hacia abajo. El agua infiltrada a través de las grietas de extensión, progresa en profundidad hasta alcanzar el contacto con el nivel masivo que actúa como nivel 

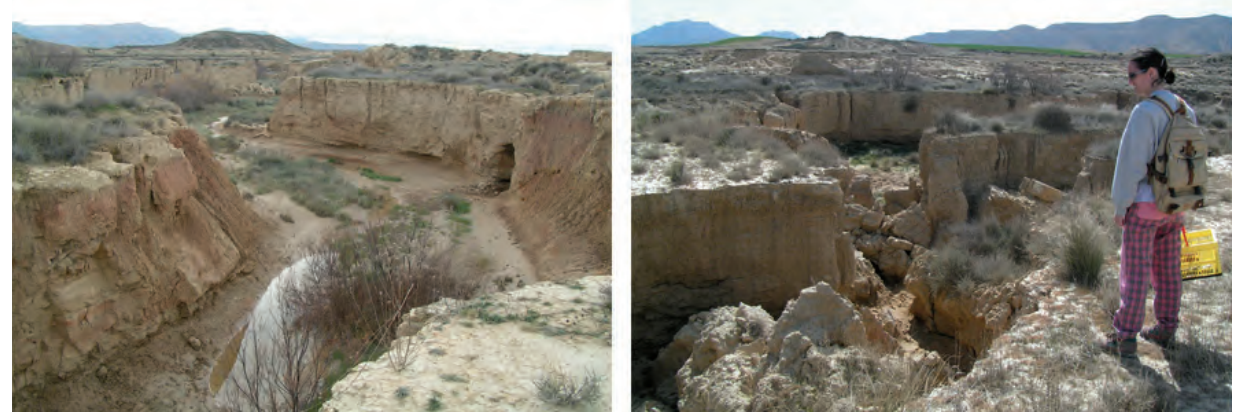

Figura 5. A) Vista general del barranco principal del área de estudio. En el margen derecho se puede observar un puente natural formado en el outlet de un gran pipe. B) Detalle de ese pipe con el puente natural colapsado. La imagen está tomada desde el margen del pipe y se observa al fondo el barranco.

impermeable y se propagan siguiendo este nivel hasta alcanzar el gully. El outlet aparece colgado sobre el fondo del gully por lo que su desarrollo está condicionado por el nivel de base (Fig.4 y 5).

En las paredes verticalizadas de los gullies de grandes dimensiones también se forman pipes en nido cuando estas paredes están coronadas por niveles encostrados en superficie.

Rellenos vs. Piping. El piping se desarrolla básicamente en los niveles intermedios masivos de los materiales del relleno holoceno de la Depresión de las Bardenas Reales, aunque desde el punto de vista de las propiedades físico-química todos los niveles son susceptibles de desarrollarlo. Estos pipes que se desarrollan sobre los niveles masivos suelen tener bajas pendientes y escasos diámetros (Fig. 6 a); aunque en determinadas situaciones los diámetros pueden alcanzar la escala métrica (Fig. 6 b). Son función del patrón de agrietamiento, de la capacidad de hinchamiento y también de las variables climáticas. Su aparición está controlada por la alternancia de épocas de lluvia y épocas de sequia que controlan la formación de las gritas de retracción que facilitan la entrada de agua en el regolito y el inicio de los pipes. Son conductos circulares o con forma de ojo de cerradura. Las dimensiones oscilan entre la escala centimétrica y decimétrica.

Su presencia en los niveles laminados superior e inferior está condicionada y limitada por la potencia de los niveles masivos intercalados. Cuando la potencia es inferior a $20 \mathrm{~cm}$, no se forman. Gracia (1986) señala una relación entre la longitud y la anchura muy superior a 5. En estos materiales el principal factor limitante del piping es la presencia de niveles algales de escasa potencia intercalados en los niveles laminares superior e inferior que limitan el desarrollo en profundidad de los pipes generando conductos de escala centimétrica que siempre están sobre impuestos a la red de drenaje. Por ello, apa- 

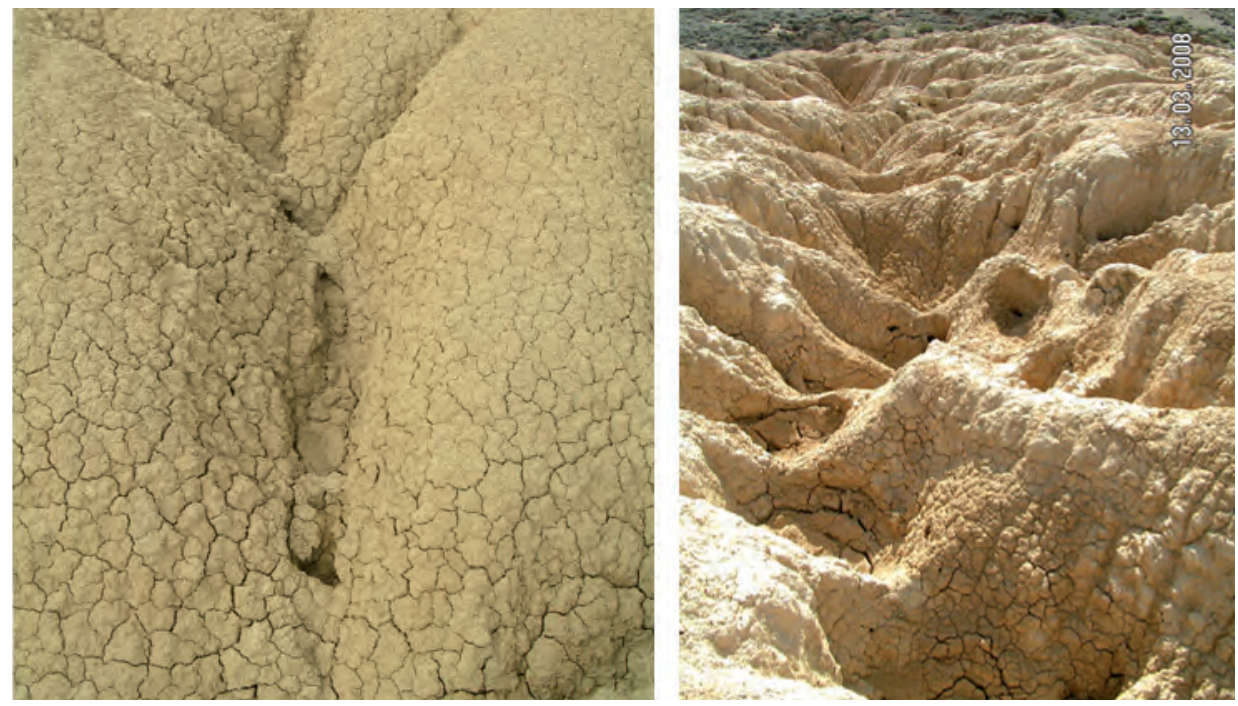

Figura 6. A) Pipe de escala decimétrica desarrollado sobre el nivel masivo intermedio. B) Pipes de dimensiones métricas en la zona de Cabecico Losado circunscritos a la red de regueros.

recen desarrollados en las zonas donde el nivel laminado superior presenta una mayor potencia y los niveles masivos intercalados tienen mayor desarrollo como es la zona del Piskerra (Fig. 7) o junto a las instalaciones militares donde el nivel laminado inferior presenta su mayor potencia.
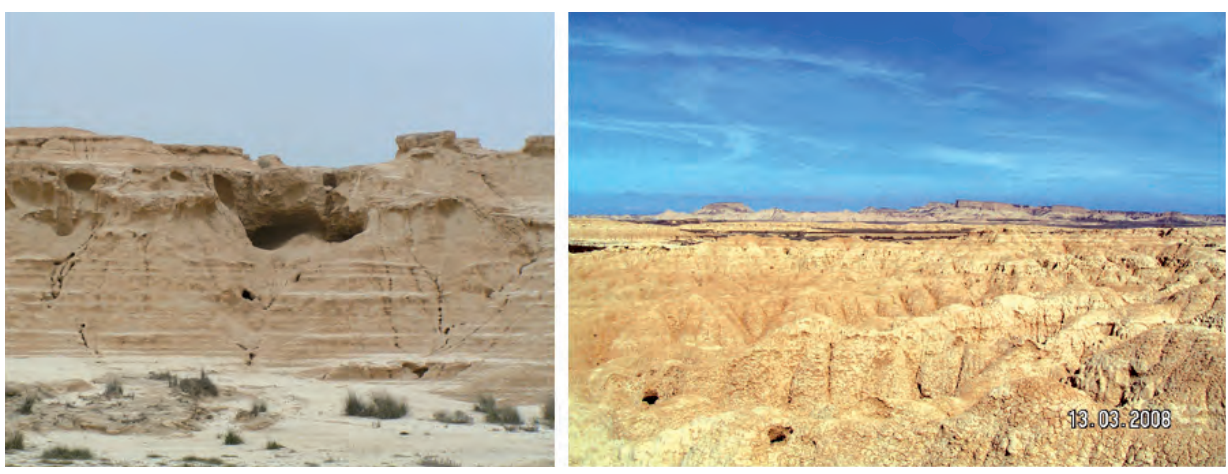

Figura 7. A) Pipe de escala centrimétrica desarrollado sobre el nivel laminado superior. B) Pipes de dimensiones decimétricas sobre el nivel laminado inferior. 
En las paredes verticalizadas de los gullies de grandes dimensiones también se forman pipes en nido cuando estas paredes están coronadas por niveles encostrados en superficie.

Caracterización del piping. El piping es el mecanismo precursor para el avance del retroceso de las cabeceras y de la ampliación del canal, siendo la densidad y magnitud de los pipes función de las dimensiones y de la distancia al margen del gully. Para caracterizar la importancia de este proceso en las Bardenas Reales se realizaron un total de cincuenta medidas de pipes de tres zonas diferentes. Como se ha señalado se han medido la anchura, longitud, profundidad de cada uno de los pipes, así como la distancia al vecino más próximo y la distancia al gully. También se midió la altura relativa del outlet sobre el fondo del gully, la potencia del nivel sobre el que se desarrollan y el tipo de grietas precursoras.

Para comprobar la relación existente entre las dimensiones de los pipes y la distancia al gully y la potencia del mismo se han representado cada una de estas variables, diámetro y superficie, frente a la profundidad de la misma y la distancia al gully. Los resultados muestran como se definen dos grupos de pipes diferentes. Esta división está relacionada con el tipo de mecanismo precursor del pipe. En el primer caso, se trata de pipes formados por grietas de tracción y procesos de tunnelling. Se trata de pipes con diámetros de diferente tamaño y que presentan distancias peque-

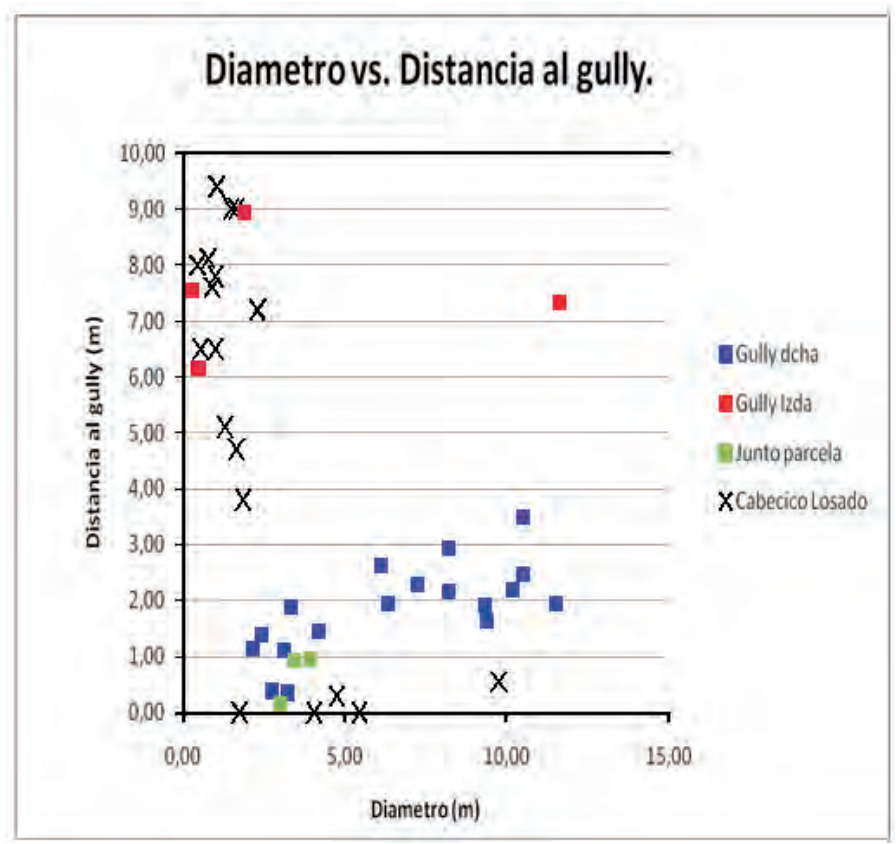

Figura 8. Relación entre el diametro y la distancia al gully de los pipes medidos en las diferentes zonas estudiadas. 
ñas respecto al margen del gully (Fig.8). El segundo tipo son pipes de pequeñas dimensiones que muestran una evolución independiente de la distancia al gully que actúa de colector local (Fig.8).

\section{Conclusiones}

La dimensión y magnitud de los pipes analizados, muestran una clara relación entre la potencia del nivel, la pendiente y el gradiente hidráulico.

En los rellenos el principal factor limitante es la potencia pues los niveles laminares inferiores no facilitan la continuidad en profundidad del proceso debido al elevado número de discontinuidades. Los pipes son de escala centimétrica y siempre están en relación con la red de regueros.

En los gullies aparecen asociados a los márgenes y son uno de los principales mecanismos de crecimiento de los mismos, estando ligados al retroceso de las cabeceras. El tamaño y diámetro de los pipes es función directa de las dimensiones del gully e inversamente proporcional a la proximidad al borde.

\section{Referencias bibliográficas}

BRYAN, R.B., JONES, J.A.A., (1997). The significance of soil piping processes: inventory and prospect. Geomorphology, 20: 209-218.

Desir, G., Marín, C., (2006). Evolución y procesos de erosión en gullies. Bardenas Reales (Navarra). España. En: Geomorfología y Territorio (Pérez-Alberti, A., LópezBedoya, J., Eds.), Sociedad Española de Geomorfología. Universidad de Santiago de Compostela.

MARíN, C., Desir, G., (2004). Influencia de las propiedades físico-químicas del regolito en los procesos de erosión. Bardenas Reales (Navarra). En: Riesgos Naturales y Antrópicos en Geomorfología (Benito, G., Díez Herrero, A., Eds.), Sociedad Española de Geomorfología. Madrid.

Desir, G., MARÍN, C., Guerrero, J., (2005). Badlands and Talus Flatirons in the Bardenas Reales Region. Field Trip Guide B3. VI International Conference on Geomorphology. Zaragoza.

FAUlKner, H., SpIVEy, D., AleXANDER, R., (2000). The role of some site geochemical processes in the development and stabilisation of three badland sites in Almería, southern Spain. Geomorphology: 35, 87-99. 
GRACIA, F.J., (1986). Geomorfología de las Bardenas Orientales. Tesis de Licenciatura. Universidad de Zaragoza

Gutiérrez, M., Sancho, C., Desir, G., Sirvent, J., Benito, G., Calvo, A., (1995). Erosión Hídrica en Terrenos Arcillosos y Yesíferos de la Depresión del Ebro. Ministerio de Agricultura, Pesca y Alimentación. Universidad de Zaragoza, 389 pp., Zaragoza.

JONES, J.A.A., (1981). The nature of soil piping: a review of research. British Geomorphological Research Group Monograph Series 3. Geobooks, Norwich. 1015

Martínez-Casasnovas, J.A., Ramos, M.C., Poesen, J., (2004). Assessement of sidewall erosion in large gullies using multi-temporal DEMs and logistic regression analysis. Geomorphology, 58, 305-321. 